\title{
State of the journal: 2019
}

\section{Edward E. Lawson ${ }^{1}$}

Received: 17 December 2018 / Accepted: 18 December 2018

(c) Springer Nature America, Inc. 2019

Periodically, I have written a "State of the Journal" editorial to inform our readers of the major activities of the journal and to publically thank our external reviewers for their efforts in refereeing many of the manuscripts submitted for consideration of publication. The reviewers remain anonymous to individual manuscript authors, but this public listing is our way of highlighting their importance in maintaining the journal's excellence and of individually thanking them for their efforts.

On December 31, I will retire as the Editor in Chief and be replaced by Patrick Gallagher from Yale. I have served as the Editor in Chief since 2001 and have enjoyed the role greatly. Dr. Gallagher was chosen after an international search by a committee consisting of representatives from our two sponsoring professional societies, the publisher, and myself. Dr. Gallagher is Professor of Pediatrics, Genetics and Pathology, Yale. He regularly attends Neonatal Intensive Care Unit (NICU) and is recognized as an outstanding clinician and teacher. He has published over 200 peer-reviewed articles largely in fetal and neonatal aspects of hematology and genetics. He supervises an NIHfunded research laboratory at Yale focusing primarily on genetics, genomics, and developmental biology of inherited blood disorders. He also participates in translational studies of infectious diseases of the neonate and various quality improvement initiatives in the NICU. He teaches trainees at all levels, from undergraduate, medical and graduate students to senior postdoctoral fellows and sabbatical faculty. I am enthusiastic about his appointment and see it as the next step in advancing the journal.

The progress of the journal has been largely due to the strong support by our contributors. Compared with 2001, the annual number of new articles received in the journal

Edward E. Lawson

elawson1@jhmi.edu

1 Johns Hopkins School of Medicine, 1800 Orleans Street, Mailstop 8-South, Baltimore, MD 21287, USA has quadrupled. Several years after I began, the number of issues published per year increased from 8 to 12. By 2017 we doubled the number of original articles per issue compared with 2011 despite discontinuing the popular case report series, which in 2014 had contributed to $25 \%$ of all the new articles received. Our Impact Factor varies from year to year but generally puts us around the top third of journals in the pediatric category. The Eigenfactor (similar to the Impact Factor, but adjusted for the impact of the citing journals) for 2017 is tied for \#18, ranking among the 124 pediatric journals evaluated by Web of Science. We certainly appreciate the increasing support from all our contributors who continue to provide high-quality manuscripts.

One aspect of past editorials has been a brief discussion of our responses to the "hot" issues relating to the process of publishing original research. In the last editorial (2016), I discussed about bogus or fabricated reviews of articles and aspects of paying for open access (OA) as it relates to the Journal of Perinatology. I tried to assure our readers that we have safeguards to prevent fabricated reviews and that OA had no role in the editorial decision process. During the past few years, several aspects of plagiarism have occurred that I believe need the attention of our readership so that they may avoid those issues.

Plagiarism is defined as replicating the works by others, without proper attribution of that work. That replication may also be of one's own work in which it is described as "self plagiarism". The problem with plagiarism is that it is considered intellectual dishonesty (an ethical issue) or copyright violation (a legal issue). In either case it is to be avoided at all costs. In scientific reporting, intellectual dishonesty can be career ending because it demonstrates that a researcher's scientific results are not dependable, potentially much more significant than violating copyright laws. In order to detect the possibility of duplications in manuscripts about to be published, in the final stage of being accepted for publication, each manuscript is sent through an on-line computer program ("CrossCheck/Ithenticate") that compares the manuscript to all the material that has been 
published and placed onto widely distributed electronic resources (e.g., the worldwide web). CrossCheck highlights short and long sequences of duplicated words that it finds in that database and reports a percentage of words in the text of the manuscript being considered that appear to be duplicated or paraphrased. When we get the report back from CrossCheck an editor manually reviews all manuscripts that score above 25\% "duplications". Mostly, the identified text consists of a few words that are frequently "stock phrases", institution names, etc. Stock phrases include things like disclaimers or commonly used descriptors (such as "Supported by the National Institutes of Health and the Eunice Kennedy Shriver National Institute of Child Health ..." or "...defined as presence of grade III or IV intracranial hemorrhage or cystic periventricular leukomalacia...."). None of these are considered duplicates because they are so common. However, when multiple sentences or even whole paragraphs are identified then we investigate further. Recently, CrossCheck identified several manuscripts that were examples of clear plagiarism. One was an attempt by a research group to publish a fifth article that used tables and text in each of the four prior articles. Another example was a manuscript that had several duplicated paragraphs and a large table with identical results from an unreferenced publication. These were two clear examples of intentional plagiarism. We respond to such examples using processes defined by the Committee on Publication Ethics [1].

However, we have now also seen examples of three ways in which authors, perhaps unintentionally, plagiarize the work of themselves or others. First is a research group repeating the exact description of methods used in previous research publications from their group. Many people, including me, consider this a "minor sin" as there are only so many ways one can restate the same techniques. However, the original verbiage is likely under copyright, and thus this is a legal issue. I prefer simply that authors using same or similar methods should simply state "We have described the methods earlier (ref no.)". That can be followed by a brief description and/or simply the aspects unique to the present report. That saves print space and cost, and the readers can find the actual methods if they need.

A second insidious, but possibly accidental, method of plagiarizing your work or that of others is when writing a manuscript (we have seen this many times especially in reviews) one copies and pastes a paragraph or two from a report fully, intending to rewrite it later. However, when revising the manuscript the copied words are so well written (after all they were published!) that one overlooks the needed alterations. The message is never copy and paste published material into your work that you intend to publish. Even paraphrasing such paragraphs by deleting or replacing a few words is identified by CrossCheck.

The above two examples of preventable plagiarism should be known to experienced authors. However, we identified recently a third way in which a naive author was ensnared into an unintentional duplication of an entire manuscript. This happened when he was solicited by one of the new OA journals to submit an article. The author emailed in a manuscript and it was accepted without further review. The manuscript was processed and published and then the publisher demanded payment. Unaware of the publication of the manuscript, the author refused payment and requested that the paper not be published. Having no further correspondence (except further bills) they assumed it was not published and sent the manuscript to Journal of Perinatology for evaluation. Our reviewers felt that it was a fine manuscript and advised publication with suggested manuscript changes. On resubmission, we sent the revised manuscript through CrossCheck and it received an $80 \%$ duplication score. We rejected the manuscript and, following Committee on Publication Ethics recommendations [1], requested the institution to investigate the apparent self-plagiarism. The institutional investigation identified the above sequence of events and concluded that the original publication was unintended by the authors. This could have been avoided by the authors assuring that they were submitting via a formal and identified process that they understood.

Publisher's note: Springer Nature remains neutral with regard to jurisdictional claims in published maps and institutional affiliations.

\section{References}

1. Committee on Publication Ethics. COPE statement on "How should editors respond to plagiarism?" http://publicationethics.org/files/ u7141/Discussion\%20document_0.pdf. 


\section{Journal of Perinatology: completed manuscript reviews January 2016 through November 2018}

\author{
Ahmed M Abbas \\ Jodi Abbott \\ Ebtesam M Abdalla \\ Carolyn L Abitbol \\ Smail Acimi \\ David H Adamkin \\ Anne Ades \\ Vincent Adolph \\ Zubair H Aghai \\ Charles Ahlfors \\ Kaashif Ahmad \\ Abimbola Aina-Mumuney \\ James Akula \\ Mohamad Al-Hosni \\ Kurt H Albertine \\ Sofia Aliaga \\ Gabriel Altit \\ Hany Z Aly \\ Gil Amarilyo \\ Sanjiv B Amin \\ Kimberly Amundson \\ Zhiqiang An \\ Frank W J Anderson \\ Michael Anderson \\ Harry Applebaum \\ Adi Aran \\ José Arellano-Galindo \\ Ronald Lee Ariagno \\ Mark Arkovitz \\ Cody C Arnold \\ Shmuel Arnon \\ Ami Ashar \\ Kristin Ashford \\ Gabi Gshkenazi \\ Joshua Attridge \\ Susan W Aucott \\ Topun Austin \\ Laura Avagliano \\ Christoph Bührer \\ Carl H Backes, Jr. \\ Michelle Baczynski \\ Henrietta Bada \\ Rebecca N Baergen \\ Willem Baerts \\ Jennifer Bailit \\ Monika Bajaj \\ Maya Balakrishnan \\ Jerasimos Ballas \\ Eduardo Bancalari \\ Maskit Bar-Meir \\ Benjamin Bar-Oz \\ Rosilu Ferreira Barbosa \\ Maree Barnes \\ Keith J Barrington \\ Karsten Bartels \\ Mir Abdul Basir \\ W Thomas Bass \\ Haim Bassan \\ Sriparna Basu \\ Beau Batton \\ Stephen Baumgart
}

Kelley Baumgartel

Moraye Bear

Cynthia F Bearer

Steven Beaudry

Jill C Beck

Alan Bedrick

Mandy B Belfort

Jaques Belik

Marc Beltempo

Melania Bembea

Efrat Ben-Shalom

George Jesse Bender

William E Benitz

Michael D Benson

Ron Benzie

Andrew Berenz

Marie Berg

Richard Berger

Cheston Berlin

Loren Berman

Anita Bhandari

Vineet Bhandari

Ramachandra Bhat

Jatinder Bhatia

Vijayalakshmi Bhatia

Shazia Bhombal

Vinod K Bhutani

Margarita Bidegain

Alona Bin-Nun

Manoj Biniwale

Cahit Birdir

Howard J Birenbaum

Nese Biyikli

Matthew Bizzarro

Knut Bjuland

Joseph M Bliss

Steven M Block

Barry T Bloom

Yair Josh Blumenfeld

Kajsa Bohlin

Sonia L Bonifacio

Willem de Boode

Arend F Bos

Thangamadhan Bosemani

Renee D Boss

Francesc Botet

Farid Boubred

Isabelle Boucoiran

Ghada Bourjeily

Theonia Boyd

Annelee C Boyle

Kenneth M Brady

Mary Brandt

Sharron Bransburg Zabary

Pablo Hernán Brener

Brian Bridges

Luc P Brion

John R Britton

Brian Brocato

Ruben Bromiker

Itzhak Brook
Laura Davidson Brown

Emmanuel Bujold

Dale Bull

Giuseppe Buonocore

David James Burchfield

Irina Burd

Bryan Burke

Jennifer Burnsed

Heather H Burris

Richard Burwick

Alexander Butwick

Darlene Anne Calhoun

Deborah Campbell

Michael Caplan

Alison Carey

Waldemar A Carlo

J Bryan Carmody

Patrick Douglas Carroll

Brian Carter

Ebony Boyce Carter

$\mathrm{K}$ Cashen

William J Cashore

Andrea Cassidy-Bushrow

Anita J Catlin

Aaron B Caughey

Ibrahim Ilker Cetin

Hala Chaaban

Rosette Chakkalakal

Lina F Chalak

Sophelia Chan

Praveen Chandrasekharan

Jen Jen Chang

Ira J Chasnoff

Raul Chavez-Valdez

Dafang Chen

Dongbao Chen

Steven Chernausek

Frank Chervenak

Nancy C Chescheir

Po-Yin Cheung

Christian A Chisholm

Karen-Marie Chisholm

Ramen H Chmait

Claire Chougnet

Nitin Shashikant Chouthai

Robert D Christensen

Helen Christou

Michael Ciccolo

Mark Allen Clapp

David A Clark

Reese Hunter Clark

Steven Clark

Nelson Claure

Ronald Ian Clyman

Susan Coffey Zern

Judy Slome Cohain

Bernard Cohen

W R Cohen

Vanessa Cole

Jerry Coleman

Sally Collins
Christopher A Collura

Josef Cortez

Erich Cosmi

Celia Costa

C Michael Cotten

María L Couce

Xanthi Couroucli

Sherry Courtney

Callie Cox Bauer

Mauro Cozzolino

Alexa Kanwit Craig

Jane Crosson

Milenka Cuevas Guaman

Christy L Cummings

James John Cummings

Alain Cuna

Amy Cunningham

Karen D'Apolito

Saakje da Costa

Rita Dadiz

Kay Daniels

Valery A Danilack

Abhik Das

Jonathan Davis

Natalie Louise Davis

Adekunle Dawodu

Maria F de Almeida

Maria De Ocampo

Linda $\mathrm{S}$ de Vries

Michele DeGrazia

Marloes Dekker Nitert

Thomas Deloughery

Stephen Daniel DeMeo

Eugene M Dempsey

Jeffrey Morgan Denney

Erin M Denney-Koelsch

Ashok Deorari

Koert DeWaal

Ramasubbareddy Dhanireddy

Juliann Di Fiore

Elena Díaz

Janet DiPietro

Pamela K Donohue

Jennifer Duchon

William Patrick Duff

Dmitry Dukhovny

Vikram Dumpa

Tara Lynn Dupont

Manuel Durand

Sourabh Dutta

Margaret Dziadosz

Brownsyne T Edmonds

Deborah Ehrenthal

Eric C Eichenwald

Arthur I Eidelman

Nathalie El Ters

Afif Faisal EL-Khuffash

Walid El-Naggar

Yasser Yehia El-Sayed

Barbara Engelhardt

William A Engle 
Carey Eppes

Stephen Eppes

Beth Epstein

Ebru Ergenekon

Juan-Sanchez Esteban

S Eventov-Friedman

Andrew Ewer

Fabien Eyal

Karen Fairchild

Roger G Faix

Ale Familiari

Jonathan Fanaroff

Jennifer Lynn Fang

Florian Faschingbauer

Aviva Fatal

Maisa N Feghali

Erika F Fernandez

Neil N Finer

Jennifer Fitzgerald

April Danielle Fogleman

Elizabeth Ellen Foglia

Alonzo T Folger

Rafael Fonseca

Sotirios Fouzas

Regina Fragneto

Gary Francis

Gary Freed

Yvonne Freer

Heather M French

Heather Frey

Alex Friedman

Deborah M Friedman

Stephanie Fritz

Jessica Teresa Fry

Alan M Fujii

Kazumichi Fujioka

Janusz Gadzinowski

Patrick G Gallagher

Maria Gallo

Prakash S Gambhir

Dawn Gano

Chalres Garabedian

Arlene Garingo

Jeffery Scott Garland

Sandra S Garner

Mark Gaylord

Silvia Gazzin

Cara A Geary

Polina Gelfer

Stacie Geller

Jeffrey S Gerdes

Ira Gewolb

Andrew William Gill

Shannon L Gillespie

Guillermina Girardi

Camilla Gizzi

Chris J Glantz

Hannah C Glass

Penny Glass

Christina Glick

William C Golden

Jay P Goldsmith

Mitchell Reid Goldstein

Neal D Goldstein

Ricki Friedman Goldstein

Sergio G Golombek
Fabiana Gonçalves

Cynthia L Gong

Jenn Gonya

Misty Good

Phillip V Gordon

Jeffrey Gould

Rathinaswamy Govindan

Ernest Graham

Marybeth Grant-Beuttler

Pauline D Graziano

Rachel G Greenberg

Michelle M Greene

Anne Greenough

Frank Greer

Gorm Greisen

Ian Griffin

Ursula Guillén

Ronnie Guillet

Ruth Guinsburg

Neslihan Gungor

Ashish O Gupta

Munish Gupta

Samir Gupta

David M Haas

David Hackney

Eran Hadar

James I Hagadorn

Safwan Halabi

Richard Hall

Sue L Hall

Rana Hamdy

Maureen Hamel

Cathy Hammerman

Shannon Hamrick

Corrine Hanson

Jane E Harding

Hassan Harirah

Lorie M Harper

John V Hartline

Leon Dupree Hatch, III

Thomas Havranek

Marlyse Haward

William Hay

Julia Heck

William Hersh

Eric Herzog

Micah Hester

Kent D Heyborne

Eiji Hirakawa

Timmy Ho

Thomas Hoehn

M Camille Hoffman

Matthew K Hoffman

Suma Bhat Hoffman

Julie Hofheimer

Diane Holditch-Davis

Michael F Holick

Margaret L Holland

Bruce W Hollis

Liisa Holsti

Thomas Hooven

Eric Horowitz

Guoying Huang

Jeryl Huckaby

Mark L Hudak

Elizabeth Hurrion
Naveed Hussain

Sunah Susan Hwang

Jon Hyett

Michael T Hynan

Nicholas Illsley

Terrie E Inder

Maribeth Inturrisi

Saleem Islam

Susan E Jacobs

Denise Jacobson

Sudarshan R Jadcherla

Amish Jain

Siddharth Jain

Sunil Jain

Sushil K Jain

Viral G Jain

Jan Janota

Lauren M Jansson

Annie Janvier

Angie Jelin

Laura L Jelliffe-Pawlowski

Erik Allen Jensen

Priscilla Joe

Clark Timothy Johnson

Celeste Johnston

Lindsay C Johnston

Abbey Jones

Ryan Jones

Cassandra Josephson

Ashley Juhl

Kai König

Joseph Kaempf

Anjali J Kaimal

Venkatakrishna Kakkilaya

Mary Kamb

Jegan Kandasamy

Vishal Kapadia

Michael Kaplan

M Gary Karlowicz

Ericalyn Kasdorf

Anup C Katheria

David Kaufman

Ann Kavanaugh-McHugh

A Kavitha

Masahiko Kawai

William Keenan

Folasade Kehinde

Sven Kehl

Ann Kellams

Stephen Kempley

Carole Kenner

Martin Keszler

Asma Khalil

Alhossain Khkhalafallah

Stephen D Kicklighter

Helle Kieler

Howard W Kilbride

Katarzyna Kilis-Pstrusińka

Sohye Kim

Brian Christopher King

D Kiortsis

Russell S Kirby

Yasmine Kirkorian

Haresh Kirpalani

Monica Kleinman

Pamela Jean Kling
Claus Klingenberg

Martin Kluckow

Robin B Knobel

Prabhakar Kocherlakota

Lazaros Kochilas

THHG Koh

Alexander Kon

Winston Koo

Loretta Kopelman

Robert James Kopotic

Robert Koppel

Steven Korzeniewski

Milton Kotelchuck

Courtney Kraus

Amir Kugelman

Stephanie K Kukora

Ajay Kumar

Mirjam Kunze

Venkata S Kuppala

Dalibor Kurepa

Satoshi Kusuda

Edmund La Gamma

John Ladino

Harry N Lafeber

Shabnam Lainwala

Satyan Lakshminrusimha

Reeta Lampinen

Wannasiri Lapcharoensap

Abbot R Laptook

Mariam Susan Latuga

Chantal Lau

Matthew Laughon

Naomi Laventhal

Adrian Lavery

Anne Lavoie

Shelly Lawrence

Edward E Lawson

Ben Lee

Henry C Lee

Maureen Lefton-Greif

Liisa Lehtonen

Monica E Lemmon

Tina A Leone

Mary Leppert

Lisa D Levine

Judy Levison

Orly Levit

Philip Thaler Levy

Adam Lewandowski

David Lewis

David Lewis MD

Edward Liechty

C Lindblad-Wollmann

Ita Litmanovitz

Sarah E Little

William Frank Liu

Robert Locke

Abhay Kumar Lodha

John Wells Logan

Enrico Lopriore

Diane Estella Lorant

Scott Andrew Lorch

Jeffrey Loughead

Mary Lovegreen

Harold N Lovvorn, III “

Jean R Lowe 
Kera Luckritz

Deirdre Judith Lyell

Corrie Macdonald-Wallis

Everett F Magann

John D Mahan

Akhil Maheshwari

Volker Mai

M Jeffrey Maisels

Nathalie Linda Maitre

Imad Makhoul

Shadi Malaeb

Daniel Malleske

Michael Howard Malloy

Mark C Mammel

Nicasio Mancini

Dror Mandel

George Mandy

Brett James Manley

Paul Mann

Melanie Manning

Krishelle L Marc-Aurele

Neil Marlow

Ronella Marom

Jill Lamanna Maron

Peter B Marschik

Nicole Marshall

Gilbert Ira Martin

Richard J Martin

Gerald Marx

An N Massaro

Francesco Massart

Mona Massoud

Amit M Mathur

Dana Matthews

Michael McCulloch

Thomas McElrath

Steven McElroy

Cynthia McEvoy

Jenny McFarlane

Jane E McGowan

Rachel Therese McGrath

Jennifer McGuirl

Patrick J McNamara

Emily M McNellis

Robert McVie

Barbara Medoff-Cooper Cristian Meghea

Paula P Meier

Samuel Menahem

Judith S Mercer

Stephanie L Merhar

Thurman Allen Merritt

Karen K Mestan

Andrew Meyer

Jeffrey M Meyers

Eedy Mezer

Ulrike Mietzsch

Lukas Peter Mileder

Jan Miletin

Marvin Elliott Miller

Francis Mimouni

Jonathan Philip Mintzer

Dan Miron

Mohamed A Mohamed

Deepika Mohan

Mahendranath Moharir
James Moore

Sam Moore

Tiffany A Moore

Karen H Morin

Dean Morrell

John Morrison

John C Morrison

Patrice Gilbert Morville Ahmed

Moussa

Michael E Msall

Dennis Mujsce

Amit Mukerji

Sagori Mukhopadhyay

Sarah Mulkey

Luke Charles Mullany

Upender Krishen Munshi

David Munson

Jonathan Muraskas

Deirdre M Murray

Jeffrey C Murray

Karna Murthy

Kinga Musiał

Ali M Nadroo

Craig Nankervis

Vivek Narendran

Hannah Nathan

Howard Needelman

Leif D Nelin

David Bryan Nelson

Josef Neu

Jaana Nevalainen

Dennis Nielson

Susan Niermeyer

Amiram Nir

Itamar Nitzan

Mary L Nock

Lawrence Nogee

Constance Noguchi

Shahab Noori

Mary Norton

Donald M Null

Sushma Nuthakki

Louise M O'Brien

Betsy O'Donnell

Keelin O'Donoghue

Donough O'Donovan

Joyce O'Shea

Thomas Michael O'Shea

Perrie O'Tierney-Ginn

Robin K Ohls

Bryan L Ohning

Hidenobu Ohta

Felix Amaibi Okah

Irene Olsen

Alice Ordean

Kelly Orzechowski

Matt Oster

Betsy Ostrander

Tulin Ozcans

Bettina Paek

Jessica Page

Anna Palatnik

Marjorie Meyer Palmer

Richard Parad

John James Paris

Helena Parkington
Aloka L Patel

Neil Patel

Pinkal Patel

Ravi Mangal Patel

Sunil Pati

Heather Patisaul

Sanjay Patole

Kamakshya P Patra

Stephen W Patrick

Philippos Patsalis

David Andrew Paul

Megan Elizabeth Paulsen

Menelaos Pavlou

Antoine Payot

Stephen Pearlman

Elizabeth A Pector

Max Perlman

Milan Perovic

Maryanne T Perrin

Serafina Perrone

Thomas Peterman

Esben Petersen

Anna Petrova

Raylene M Phillips

Alan Peter Picarillo

Jean-Charles Picaud

Carlo Pietrasanta

De-Ann M Pillers

Halit Pinar

Roberta Pineda

Joaquim M B Pinheiro

Francesco Pisani

Michael Pitt

Chiara Poggi

Brenda Poindexter

Sruthi Ratna Polavarapu

Jeffrey Pomerance

Michael A Posencheg

Richard James Powers

Arun Pramanik

Sarah Price

Wayne Price

Karen Pridham

Gabriella Pridjian

Jochen Profit

Gloria S Pryhuber

Karen Marie Puopolo

DeWayne Pursley

Louisa Pyle

Graham E Quinn

Kristiina Rönö

Heike Rabe

Paula G Radmacher

Talkad S Raghuveer

Aiman Rahmani

Rangasamy Ramanathan

Gladys A Ramos

Aadia Rana

Tara Randis

Sukhvinder Ranu

Rakesh Rao

Nicholas JW Rattray

Mehul Raval

Munmun Rawat

Andrei Rebarber

Raymond Redline
Jeff Reese

Brian Reichman

Shimion Reif

Michael Repka

Heiko Reutter

Rodolfo Rey

Eric W Reynolds

Wade Rich

Erin Richardson

Jennifer Ridgeway

Peter C Rimensberger

Steven Ringer

Daniel T Robinson

Rasmus Rogvi

Mario Augusto Rojas

Claudia Roll

Asaph Rolnitsky

Charles R Rosenfeld

Warren Rosenfeld

Barak Rosenn

Michael G Ross

Shannon Ross

Beverly Rossman

Edwige Roy

Paul Rozance

Henry J Rozycki

Laura Helena Rubinos

Rita Marie Ryan

Kelli Ryckman

George Saade

Azif Safarulla

Amber Samuel

Anna Sandstrom

Meera N Sankar

Guiherme M Sant'Anna

Magda Sanz-Cortez

Subrata Sarkar

Ola Didrik Saugstad

Renate Savich

Taylor Sawyer

John Scafidi

Melissa Scala

Richard J Schanler

Robert L Schelonka

Michael S Schimmel

Elizabeth Schlaudecker

Katharina Schmitt

Georg M Schmolzer

Uwe Schneider

Sarah Schott

Michael D Schreiber

Joseph Schulman

David L Schutzman

Theodore C Sectish

Jeffrey Lewis Segar

Arvind Sehgal

Kris C Sekar

Hen Sela

Brian Shaffer

Birju A Shah

Prakesh S Shah

Seetha A Shankaran

Steven M Shapiro

Meghan Sharp

Jayant P Shenai

Edward Shepherd 
Mardelle Shepley

Michael Parker Sherman

Eric Stuart Shinwell

Philip A Shlossman

Billie Lou Short

Bijan Siassi

Asha Siddappa

Jean M Silvestri

Umberto Simeoni

Robert A Sinkin

Thitinart Sithisarn

Erik D Skarsgard

Jonathan Slaughter

William B Slayton

Marcela Smid

P Brian Smith

Gregory Sokol

Martha Sola-Visner

Sadaf Soleymani

Roger Soll

Moeun Son

Clara Song

Teresa N Sparks

Diane Lynn Spatz

Michael L Spear

Michael E Speer

David Spielberg

Joseph A Spinnato

Alicia Spittle

Shanthy Sridhar

Sindhu K Srinivas

Pinchi Srinivasan

Annetine Staff

Brian K Stansfield

Theodora A Stavroudis

John Stefano

Zina Steinberg

Lisa M Stellwagen

Olof Stephansson

Valerie T Stewart

Eyal Steyer

Oliver Stirrup
Brian Stone

Barbara Stonestreet

Marya L Strand

Lane Strathearn

Tzipora Strauss

Emily Su

KN Siva Subramanian

John Sullivan

Gautham K Suresh

Piia Helena Suursalmi

Jonathan R Swanson

Anne Synnes

Ajay J Talati

Peter Tamas

David Tanaka

Kristin Tanney

William Tarnow-Mordi

Sarah N Taylor

Arjan Te Pas

Helena Teede

Robert Glenn Tefft

Kadir Ş Tekgündüz

Lorene A Temming

Jeffrey Testani

Bradley Terrill Thach

Niranjan Thomas

Christopher Timan

Yuk Joseph Ting

Pierre Tissieres

Tsukasa Torigoe

Dena Towner

Stephanie Ellen Trend

Karli Treyvaud

Jennifer K Trittmann

Kuojen Tsao

Amy Turitz

Deborah Tuttle

Marcel Twickler

Michael Uhing

E Ramsey Unal

Mark A Underwood

Kim Updegrove
Cherry Uy

Frank Van Bel

C Van den Akker

Michael A van Manen

Krisa Page Van Meurs

John A Vanchiere

Deborah VanderVeen

Heili Varendi

Yvonne Eleanor Vaucher

Maximo Vento

Henrik Verder

Eduard AA Verhagen

Mandira Verma

Sourabh Verma

Marijn Jorien Vermeulen

Dharmapuri Vidyasagar

Gustavo Vilchez

Rose Marie Viscardi

Sreekanth K Viswanathan

Betty Vohr

Arastoo Vossough

Shilpa Vyas-Read

Carol L Wagner

Dan Waisman

Asnat Walfisch

Matthew Ben Wallenstein

Michele C Walsh

William Walsh

Frans J Walther

Robert M Ward

Wambui Waruingi

Jon F Watchko

Kristi L Watterberg

Hope Weiler

Gary Weiner

Rachel Weinerman

Andrea S Weintraub

Dany E Weisz

Joern-Hendrik Weitkamp

Sven Wellmann

Stephen Welty

Bjorn Westrup
Robert D White

Sara White

John A Widness

Nancy Wight

Anna-Karin Wikstrom

Benjamin Wilfond

Pierre Williot

Rodney Willoughby

Martha S Wingate

Jameel Winter

Sarah Winter

Christine Winterbourn

David D Wirtschafter

D Wisniewska-Ulfik

Thomas E Wiswell

Jakub Wojcicki

Ronald J Wong

Tricia Elaine Wright

Shu Wu

Yvonne Wu

Myra Helen Wyckoff

James Lawrence Wynn

Chuanbo Xie

Batia Yaffe

Hideto Yamada

Vivien Yap

Jennifer K Yee

Lynn M Yee

Branka Yli

Bradley A Yoder

Timothy York

Karen Cecile Young

Thomas E E Young

Noelle E Younge

Nahla Zaghloul

Santina Agnes Zanelli

Israel Zelikovic

Danielle Zerr

Hong-Bo Zhao

Xiaoqun Zheng

Ekhard E Ziegler

John Zupancic 